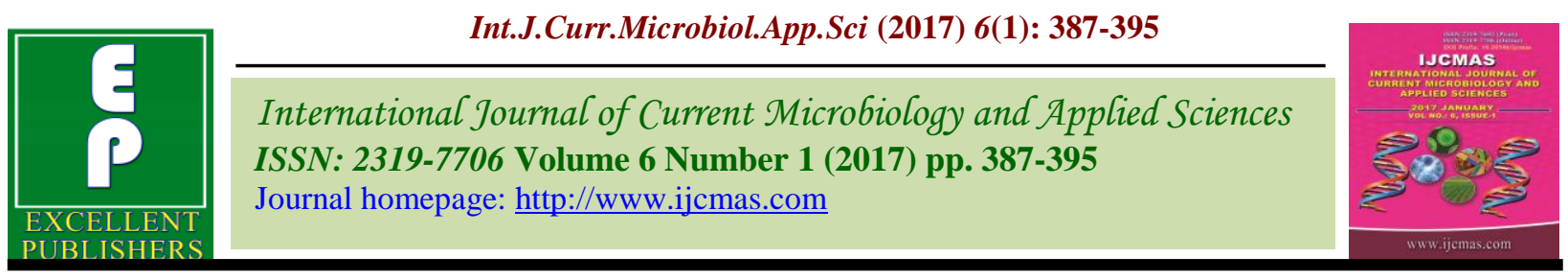

Original Research Article

http://dx.doi.org/10.20546/ijcmas.2017.601.047

\title{
A Study on the Laboratory Profiles in Dengue Viral Infection
}

\author{
Krishna Murthy Sirigadha ${ }^{{ }^{*}}$ and Md. Khaleel ${ }^{2}$ \\ ${ }^{1}$ Department of Microbiology, CAIMS Karimnagar, India \\ ${ }^{2}$ Department of Microbiology, Deccan Medical College Hyderabad, India \\ *Corresponding author
}

\section{A B S T R A C T}

The objective of the present study is 1 . To study the changing laboratory profiles in dengue viral infections. 2. To correlate these laboratory tests during the evolution of dengue fever and in assessing severity of disease. 3. To study the association of these profiles in remission of the disease. 4 . And also to study factors involved in predicting prognosis. A total number of 110 cases which were dengue IgM Seropositive (by ELISA capture method) were included in the study from Department of Microbiology, Chalmeda Anand

Keywords

Dengue,

haematological

tests, biochemical

abnormalities,

predictors of

prognosis.

\section{Article Info}

Accepted:

18 December 2016

Available Online:

10 January 2017
Rao Institute of Medical Sciences, over a period of 1 year from August 2015 to July 2016.

These cases were analysed for blood counts, platelet counts, blood urea, Serum creatinine, serum aspartate aminotransferases and alanine aminotransferases concentration. Male to female sex ratio was 1.4:1, with majority of cases in age group of 10 to 20 years. Haematological parameters studied were, haemoglobin assay, majority of patients in CD had haemoglobin values ranging from 11.1to15gms, slight variations were seen in DHF, DSS. PCV studies showed no changes in CD patients, while, more than $20 \%$ increase from baseline values was noted in DHF (12 of 21 patients) and in DSS (3of 3 patients). Lymphocytosis with reactive forms was common finding in 70 members of 110 patients, reactive forms were more in leucopenia patients. Platelets counts showed decrease in 49 of 86 patients in CD, 8members of 21 in DHF, and 2 out of 3 patients in DSS. Thrombocytopenia was marked in age group below 15years. CD and DHF had moderate thrombocytopenia, where as DSS showed marked decrease in counts. Serial follow up of platelet counts showed a dip in platelet values at day 4 to 5 ,followed by gradual recovery in 10 to 12 days.In renal function tests, 67 members in CD had normal range and only 6 members showed elevated levels. DHF showed urea in range of $10-15 \mathrm{mg} / \mathrm{dl}$ in 11 members out of 21, in DSS 2 patients had increased blood urea levels. Serum creatinine levels were slightly reduced in age group below 15 years. Liver functions tests were also altered with increase in ALT and AST levels in severe cases. Dengue Ig M Seropositive in concordance with Peripheral blood studies are of great significance along with Haematocrit values in assessment of disease, and its progression to severe forms. Also, these parameters help in indicating the prognosis of patient and course of remission.

\section{Introduction}

Dengue is the most important emerging tropical Arboviral disease in the world today. The WHO estimates 50 million dengue infections to be occurring annually and almost half the world's population lives in countries where dengue infection is Endemic (WHO, 2009). Dengue is transmitted from human to human by the bite of female mosquito Aedes aegypti, Aedes albopictus, Aedes polynesiensis and the infection with one of 
these viruses characteristically results in fever, with headache and rash as a presenting complaints. Dengue affects human beings of all age groups worldwide. The dengue fever is widely prevalent in India and all the four serotypes are found Sharma et al., (2010). Aedes aegypti is the most common vector of dengue in India, followed by A. albopictus. The diagnosis of dengue fever is carried out based on clinical, epidemiological and laboratory data. The laboratory tests includes both non specific (Blood counts, platelets count, Haematocrit, liver function tests and renal function tests) and specific tests (Viral isolation tests, nucleic acid tests and serology - ELISA for antibody detection). The non specific tests are of prognostic value and can be easily performed. Hence, these tests are commonly being implicated in monitoring the course of the disease. The spectrum of dengue disease includes -Asymptomatic cases, dengue fever, dengue hemorrhagic fever and dengue shock syndrome. Infection can be life threatening, usually when caused by secondary infection (a dengue infection in a host that has previously been infected by a dengue virus, with heterologous virus serotype) Kumar et al., (2008). The incubation period of dengue viral infection varies from 5 to 10 days, Viremia occurs about two days prior to clinical manifestations and lasts for course of 5 to 6 days of illness. The most common clinical features of dengue viral infection is high fever, followed by rash. The rash is typically macular or maculopapular, often becoming confluent and sparing small islands of normal skin. The rash is not associated with scaling or pruritus. Pervin et al., (2004), reported occurrence of rash in $33 \%$ of patients, hepatomegaly in more than $30 \%$ of the patients. Hepatomegaly is more common in patients with secondary infection and some of these may be associated with an increase in liver transaminases. Pervin et al., (2004), reported myalgia as a commonest manifestation in asymptomatic cases (84.5\% of patients), and hence requires high index of suspicion, particularly during the dengue seasonal outbreaks. Laboratory test includes Hematological profile, biochemical assays and coagulation profiles. Hematological studies shows leucopenia to be the common change. However, there are reports of mild leucocytosis at the onset of disease, with neutrophilia. The hematocrit concentration is a highly sensitive index and is of prognostic value, it should be monitored according to the days of illness. A $20 \%$ increase of hematocrit from the baseline indicates the progression of dengue fever to dengue hemorrhagic fever Kularatne (2005). Thrombocytopenia is the most common finding, and has been one of the criteria used by WHO guidelines as a potential indicator of clinical severity. The Platelet counts tend to fall over the illness course, reaching a nadir in 7 to 10 days followed by a rapid recovery response. Lower counts (less than $50 \times 10^{9} / \mathrm{L}$ ) are seen more frequently in severe disease (Muhammad umerkhan et al., 2014), further reduction in platelet counts (less than 20,000) indicates the requirement of platelet transfusions in these high risk cases, due to increased risk of bleeding. However, the correlation between thrombocytopenia and haemorrhage is weak, with lower platelet counts correlating more closely with the severity of vascular leakage in only one study. Of the biochemical parameters, the changes observed in liver function tests were more sensitive in children. The levels of serum ALT \& AST were markedly elevated, indicating severity of liver injury. These change does not have a prognostic significance, as these changes are non specific, and similar alterations in LFT are seen in any other viral infections (Srivenuita et al., 2005). In Renal function tests, Proteinuria has been detected in as high as $74 \%$ of patients with DHF (Emerson Q. Lima et al., 2007). Raise in blood urea and serum creatinine was also noted in first 7days of 
illness. The present study is aimed at studying various hematological and biochemical parameters in patients with dengue viral infections, to assess the changes in their patterns in the disease course and also their role in progression of disease.

\section{Methodology}

The present study was undertaken at Chalmeda Anand Rao Institute of Medical Sciences from August 2015 to July 2016 (1 year). All the patients with suspected dengue symptoms were initially taken up and based on WHO inclusion criteria, seropositivity, cases were screened and included into the study. The ethical clearance was obtained for the study. All serologically confirmed patients of dengue viral infection using ELISA Ig M capture method underwent detailed clinical examination and laboratory investigations. Patients were classified into classical dengue fever (CD), dengue haemorrhagic fever (DHF), and Severe dengue (SD) (or) DSS according to WHO criteria (2009).

\section{Inclusion criteria}

Case definition of dengue fever as per WHO (2009)

\section{Exclusion criteria}

1. All the cases which showed negative serology, (or) positive for other causes of fever (malaria, widal, PUO), outpatient cases were excluded from the study.

2. Patients presenting with other co morbid infections along with dengue fever were not included into the study.

3. All the other causes of thrombocytopenia were also excluded from the study.
All the Study cases were divided into two age groups to compare laboratory findings in Children to that of the Adults.

\section{Those aged 15 years or lesser than 15 ,}

2. And above 16 years of age.

Complete blood counts were carried out by automated cell counts analyzers (HPRIBA, Pentra ES 60) along with a peripheral smear on slide, which was stained with Leishman's stain, and differential counts were performed to determine the lymphocyte count, atypical lymphocytes, neutrophil count. Platelet counts were recorded and also cross checked on stained slide in each case. The counts were reviewed over a period of one to two weeks, during their hospital stay, as reduction in platelet counts is a sensitive indicator of vascular leakage and thus the disease progression. PCV was done by Wintrobes method in each case and verified with automated cell analyzer value. Follow up of cases was done whenever needed, during the period of their hospital stay. Liver function tests and renal function tests were performed photometrically using standard kits with a routine chemical analyzer. For statistical analyses of $P$ value, regression test was used, $P$ value $<0.05$ was considered significant

\section{Results and Discussion}

Out of 475 screened cases, 110 cases which were positive for dengue serology (Ig M ELSIA capture method) were taken into the study group. The results of all the 110 positive cases with clinical and laboratory data were analyzed as follows:

All the patients were categorized into 3 groups of severity, as per WHO criteria, based on symptoms and signs at the time of presentation. 
1. Classical dengue fever- total number patients were $86(78.18 \%)$, with majority of the patients falling into this group.

2. Dengue hemorrhagic fever- total number of patients 21(19.09\%).

\section{Severe Dengue - 3 patients $(2.72 \%)$.}

The total number of male patients were 65 and females 45 , male to Female patients ratio being 1.4: 1, there was a slight predominance of male

Symptoms at the time of presentation in majority of the patients was-

Fever and rash $(\mathrm{n}=96)$, malena (or) epistaxis $(n=24)$, body pains $(n=21)$, headache $(n=15)$, abdominal pain $(n=10)$, cough $(n=7)$.

\section{Haematological profile}

Haematological parameters studied included haemoglobin concentration, PCV, WBC count, platelet counts. Haematocrit Values in $\mathrm{CD}$, DHF, SD analyzed showed major variations in DHF and SD. The patients of classical dengue fever showed no variation in haematocrit values, whereas patients in DHF showed increased values in 12 members, the reason for other patients not having increase in baseline PCV could be due to early intervention and use of fluids for resuscitation in patients with hemorrhagic manifestations. And all the patients of severe dengue had elevation in haematocrit of more than $20 \%$ from baseline. PCV changes were prominent on 4th to 5th day of illness, with increase in the values, followed by gradual return to baseline by 10 to 12 days. The values of PCV increased parallel to the severity of illness.

\section{Changes in PCV Values in CD, DHF, SD}

Haemoglobin levels were in normal range in majority of patients in all the three groups. In classical dengue fever majority of adult patients $(\mathrm{n}=41)$ had $\mathrm{Hb}$ in $11-15 \mathrm{gm} \%$, and similar was the findings in children( $n=4)$.In dengue hemorrhagic fever also adults had normal range of $\mathrm{Hb} \quad(\mathrm{n}=9)$, and out of 3 patients in severe dengue group, 1 patient had $\mathrm{Hb}$ in $15-20 \mathrm{gm} \%$, 1 patient had below $10 \mathrm{gm} \%$.

Haematocrit Values in CD, DHF, SD analyzed showed major variations in DHF and SD. The patients of classical dengue fever showed no variation in haematocrit with stable serial haematocrit values, whereas patients in DHF showed increased haematocrit value in 12 members, the reason for other patients not having increase in baseline PCV could be due to early intervention and use of fluids for resuscitation in patients with haemorrhagic manifestations. And all the patients of severe dengue had elevation in haematocrit of more than $20 \%$ from baseline.

PCV changes were prominent on 4th to 5th day of illness, with increase in the values, followed by gradual return to baseline by 10 to 12 days. The values of PCV increased parallel to the severity of illness.

\section{Showing Changes In Hematocrit Values in CD, DHF and SD}

In CD $(n=86)$ all the cases had stable haematocrit, the patients of DHF total 11 patients $(52.38 \%)$ showed elevated haematocrit in range of 40-60volume\%, and only 1 case had haematocrit above $60 \mathrm{vol} \%$, but all the cases of SD had rise in haematocrit levels $(n=3)$. $P$ value obtained was $<0.001$, hence there is a positive relationship between PCV and severity of the disease.

Platelets Count: Serial evaluation of platelet count were done, and the values observed are as follows: 


\section{Cases Showing Daywise Platelet Count Variations}

Out of 86 members in CD, total number of patients with moderate Thrombocytopenia (platelet counts between 50,000 to $1,00,000$ ) were 49 , thrombocytopenia was more in age group less than 15years $(n=5)$. DHF patients had moderate thrombocytopenia $(n=9)$, while SD group showed marked thrombocytopenia with platelet counts below 50,000 $(n=2)$. There was a fall in the platelet counts till 5th day, followed by gradual rise, reaching the baseline by 10 to 12 days.

The $\mathrm{P}$ value obtained for platelet counts correlation was $<0.001(<0.05)$, suggesting a significant correlation between platelet counts and severity of illness.

\section{Renal function tests}

Renal function test studied were blood urea and serum creatinine by biochemical assays, the levels in all 3catagories are shown below. Majority of the cases in CD had blood urea levels within the range of 16 to $45 \mathrm{mg} / \mathrm{dl}$, both in children $(n=4)$ and adults $(n=66)$. The DHF category patients showed blood urea levels below $15 \mathrm{mg} / \mathrm{dl}(\mathrm{n}=9$ in adults and $\mathrm{n}=2$ in children) where as SD cases had blood urea levels more than $45 \mathrm{mg} / \mathrm{dl}$ in majority of patients(both children and adults).

\section{Variation in Serum Creatinine Levels In} CD, DHF, SD

Serum creatinine in majority of the cases in $\mathrm{CD}$ was within range of $<1.5 \mathrm{mg} / \mathrm{dl}$ in adults $(n=47)$ and in children showed lesser than $1.5 \mathrm{mg} / \mathrm{dl}(\mathrm{n}=4)$. The DHF category patients also showed creatinine levels lesser than $1.5 \mathrm{mg} / \mathrm{dl}(\mathrm{n}=2$ in children and $\mathrm{n}=13$ adults),where as SD cases had blood creatinine levels less than $1.5 \mathrm{mg} / \mathrm{dl}$ in children $(n=1)$ and normal in one patient and elevated in another patient.

\section{Liver function tests}

All the patients were tested for levels of serum Bilirubin, and only in cases showing rise in total serum Bilirubin above $2 \mathrm{mg} / \mathrm{dl}$ further investigations of AST and ALT were undertaken.

\section{Changes in Serum Bilirubin Levels In CD, DHF, SD}

Serum Bilirubin levels were normal in majority of cases, mild increase in serum Bilirubin levels were noted in $\operatorname{CD}(n=2)$, in DHF $(n=4)$ (total 7.2\%), and in SD majority showed moderately elevated serum Bilirubin levels.

\section{Levels of AST and ALT IN DHF, SD}

Mild increase in AST and ALT levels were seen in case of DHF patients, whereas, SD patients showed marked elevation in the liver enzymes.

A total of 110 Sero-positive patients were studied for laboratory profiles in which haemoglobin, WBC counts, Differential counts, PCV, platelets counts, renal function tests (blood urea and serum creatinine levels) and liver function tests (serum bilirubin, AST, ALT ) were analyzed.

Patients were categorized into classical dengue, dengue hemorrhagic fever and dengue shock syndrome as per WHO criteria (Barniol et al., 2011). Majority of the cases in the study were of classical dengue followed by dengue hemorrhagic fever, and severe dengue. These findings were consistent, and similar to other studies, which are as follows:

In the present study we have adopted terminology from recent literatures, where the patients with Dengue fever are categorized as: 
1. Classical Dengue fever

2. Dengue haemorrhagic fever--

a) Grade 1 (without warning symptoms)

b) Grade 2 ---- termed as Severe Dengue(with warning symptoms)

Most common age group affected with dengue infection, with slight excess of male population with male to female ratio of 1.4:1. This was consistent with other studies, whereas adults in the present study because paediatric age group with dengue were referred to other tertiary care hospitals and only those cases with emergency admissions into hospital were available for the study.

Haemoglobin values were in normal range in $\mathrm{CD}$ in most of the adults and children, there were no significant differences between groups. In DHF group, we observed a greater variation of $\mathrm{Hb}$ in both groups which was most evident from the 4th to 6th days with the highest values in the group of patients aged 15 years old or more. In SD group, there was a slight variation between the groups.these findings were similar to the study undertaken by (Francisca Raimunda et al., 2012).

The Haematocrit values in Classical dengue patients was less than 40 volume\%, whereas patients of Dengue hemorrhagic fever (9of 21) had Haematocrit less than 40volume\% and 11 members had increased PCV, 1 patient had Haematocrit above 60volume\%.All the patients in Severe dengue showed rise in the Haematocrit values $(\mathrm{n}=3)$ above $20 \%$ of initial baseline values, after 3 to 4 days of febrile illness period.These findings were similar to study by Trupti dongre et al., (2015).

In the cell counts analysis, White blood cell counts showed leucopenia in majority of cases with associated relative Lymphocytosis in CD, DHF, SD. Lymphocytosis was a common finding in age group of less than
$15 y e a r$ than as seen in adult age group. These findings were consistent with other studies. The patients in CD had Leucocytosis in early phase of illness developing leucopenia later on. Leucopenia was mild in DHF, than Classical Dengue and severe dengue.

In the study by Tahir jameel et al., (2012), the reactive Lymphocytosis was observed in 93\% of patients, whereas present study it was $72.2 \%$.

\section{Showing Percentage of Reactive Lymphocytes in Present and Previous Study}

In the study, Platelet counts showed decrease in number consistently with severity of case and course of illness, with maximum fall in counts on day 5 , followed by gradual rise in the numbers. Thrombocytopenia was more marked in patients of less than 15 years age. Patients of $\mathrm{CD}$ showed mild to moderate thrombocytopenia with platelet counts in 50,000 to $1,00,000 / \mathrm{cu}$. $\mathrm{mm}$ range, in DHF category, patients had moderate thrombocytopenia with platelet count in the range of 50,000 to $1,00,000$.platelet counts were markedly reduced in SD (less than 50,000). These findings were similar to studies by Jayashree et al., (2012), Muhammad et al., (2014).

\section{Comparison of Serum Creatinine}

Deranged serum urea and creatinine levels are indicative of renal dysfunction. Previously renal function alterations have not been reported in many studies, only liver function alteration has previously been reported in dengue patients, therefore the exact relationship between variation in serum creatinine in illness and remission is to be established yet. Liver function tests included serum bilirubin, AST, ALT. However, not all 
the cases were tested for AST and ALT, only in patients with elevated serum bilirubin

(greater than $2.1 \mathrm{mg} / \mathrm{dl}$ ) AST and ALT levels were assessed.

Table.1 Sex Incidence of Classical Dengue, Dengue Haemorrhagic and Severe Dengue

\begin{tabular}{|l|l|l|l|l|}
\hline Features & Classical dengue & $\begin{array}{c}\text { Dengue } \\
\text { Haemorrhagic }\end{array}$ & Severe dengue & Total \\
\hline Males & 49 & 13 & 3 & 65 \\
\hline Females & 37 & 8 & 0 & 45 \\
\hline Total & 86 & 21 & 3 & 110 \\
\hline
\end{tabular}

(CD - classical dengue fever, DH - dengue hemorrhagic fever, SD - Severe Dengue )

Symptoms at the time of presentation in majority of the patients was-

Fever and $\operatorname{rash}(n=96)$, malena (or) epistaxis $(n=24)$, body pains $(n=21)$, headache $(n=15)$, abdominal pain $(n=10)$, cough $(n=7)$.

Fig.1

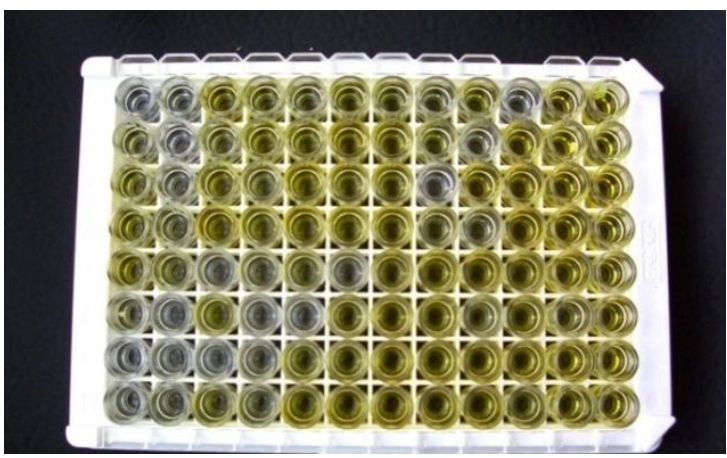

Fig.2

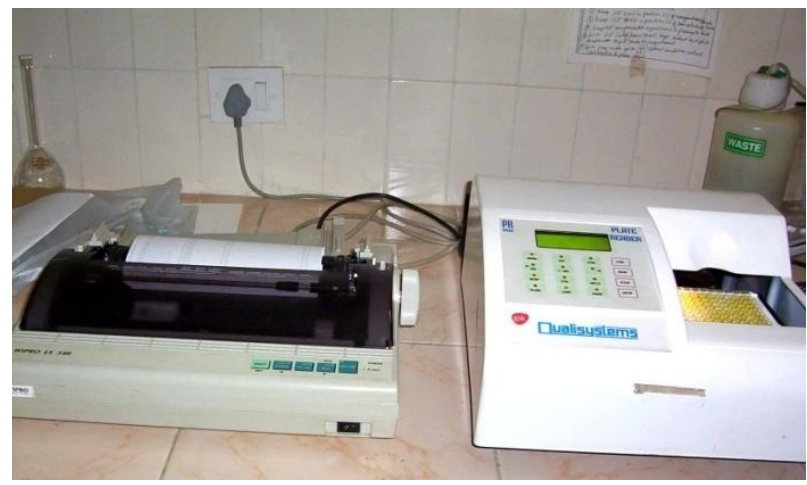

The present study data showed mild to moderate derangement in Liver Function Tests in majority of patients. The serum bilirubin levels were normal in majority of cases, only 7 patients showed elevated bilirubin levels (7.27\%), which was consistent with study by (Srivenuita et al., 2005). The magnitude of elevation of AST and ALT levels were comparable, but no preferential elevation of one of the enzymes was noted. The severity of liver injury is reflected by levels of rised AST and ALT, but, these 
markers are unlikely to be pointers of diagnosis, since liver disease can be severe in even CD M Mahmuduzaman (2011).

The ALT and ALP levels were significantly higher in DHF patients with Spontaneous bleeding than those without bleeding. None ofthe patients developed

Fulminant hepatitis $\mathrm{S}$ fadilah $\mathrm{s}$ abdul wahid (2000).

In conclusion,

1. The data from the present study shows alterations in hematological profiles, renal and liver function tests. The disease being more frequent and severe in age group 16 and older age.

2. High suspicion of dengue should be borne in patients presenting with decreased platelet counts, lymphocytosis and hemoconcentration, and they should be followed up with IgM and IgG titres.

3. The peripheral blood parameters are easily done and very useful in assessment of severity and prognosis of patient.

4. The most sensitive indicator being PCV, which helps in monitoring the progression of disease from dengue fever to dengue hemorrhagic and shock state.

5. Platelets counts serial monitoring is very important in assessment of the patient condition and preventing bleeding complications. A dip in platelet counts is seen during first five days of illness, later on the count rises to normal levels. The degree of decrease in platelet counts correlates with severity of disease and also remission status.

6. Renal involvement is also a feature of dengue viral infections, where rise in serum markers is noted, the levels of these markers correlates with severity of disease, however, these enzymes return to baseline values with remission.

7. Liver also carries the major brunt of the disease, liver enzymes are elevated in dengue, more common with SD and DHF than classical dengue cases. these values correlate with direct liver parenchymal damage levels, whether they signify the progression of disease or not is not clear yet due to relatively less number of studies on liver involvement profile.

8. To conclude, the morbidity and mortality from dengue viral infections can be significantly decreased by early laboratory diagnosis, by serial monitoring of hematological, renal and liver profiles. Among all PCV has a predictive value, which gives an indication of hemo concentration.

\section{References}

Barniol, J., Gaczkowski, R., Barbato, E.V., da Cunha, R.V., Salgado, D., Martínez, E., et al. 2011. Usefulness and applicability of the revised dengue case classification by disease: multi-centre study in 18 countries. BMC Infect. Dis., 11:106. Doi 10.1186/1471-2334-11-106, Page no: 112.

Emerson, Q., Lima, Fernanda, S., Gorayeb, Jeferson, R., Zanon, Mauricio, L. Nogueira. July 2007. Dengue haemorrhagic fever-induced acute kidney injury without hypotension, haemolysis (or) rhabdomyolysis, Nephrol. Dial. Transplant., $\quad$ Vol 22(11):. Doi: 10.1093/ndt/gfm431 Page no:33223326.

Fadilah, S., S. Abdul Wahid, Sahrir Sanusi, Mazlammohd zawawi, Raymond Azmanali. 2000. A comparison of the pattern of liver involvement in Dengue hemorrhagic fever with classic dengue 
fever, South East: Asian J. Trop. Med. Public Health, Vol: 3(2), Page no:259263.

Francisa Raimunda, F., Guerreiro Azin, et al. 2012. Dengue Profile of Hematologycal \& Biochmical Dynamics. Rev. Bras. Hematol. Hemoter., Sao Paulo. Vol 34(1) Page no: 36-41.

Jayashree, K., G.C. Manasa, P. Pallavi and G. V. Manjunath. 2011. Evaluation of Platelets as Predictive Parameters in Dengue Fever, Indian J. Hematol. S. Blood Transfus., 27(3): Doi : 101007/s 12288-011-0075-1. Page no: 127130.

Kumar, R., P. Tripathi, S. Tripathi, A. Kanodia, S. Pant, V. Venkatesh. 2008. Prevalence and Clinical Differentiation of Dengue Fever in Children in Northern India, Infect. Clin. Epidemiol. Study, Vol: 36(5), Doi: $\quad 10.1007 / \mathrm{s} 15010-008-7172-6$.

Page no: 444-449.

Mahmuduzzaman, M., Chowdhury, A.S., Ghosh, D.K., Kabir, I.M., Rahman, M.A., Ali, M.S. 2011. Serum transaminase level changes in dengue fever and its correlation with disease severity. Mymensingh Med., Vol: 20(3): Page no: 349-355.

Muhammad Umer Khan, Raima Rehman, Muhammad Gulfraz and Waqas Latif. 2014. Incidence of thrombocytopenia in seropositive dengue patients, Int. J. Med. Med. Sci., Vol: 6(4). Page no:113-116. Doi : 10.5897/ ijmms2013-1026.

Pervin, M., Tabassum, S., Md. Mobarak Ali, Mamun, K.Z., Nazul nazrul, Islam, M. 2004, Clinical and Laboratory Observations Associated with the 2000.
Dengue Outbreak in Dhaka, Bangladesh. Dengue, Bull., Vol: 28: Page no:96-106.

Sam kularatne, I.B., Gawarammana and P.R.V. Kumarasiri. 2005/ Epidemiology, clinical features, Laboratory Investigations and early Diagnosis of Dengue Fever in Adults: A descriptive study in Sri lanka, South East Asian J. Trop. Med. Public Health., Vol 36(3).Pageno:686692.

Sharma, S.K., Gautam Ahluwalia. 2010. Dengue Fever in India an overview, new Delhi. Medicine update, volume 20.Page no: 657-659.

Srivenuita, Rajesh Kashyap, Narender Krishnani. 2005. Profile of liver involvement in Dengue viral infection. The National Med. J. India, vol 18(3), P no: $127-130$

Tahir jameel, Khaleed mahmood, Ghulam mujhtaba, Nakhshab choudhary,Naeema afzal, Rubina, F.P. 2012. Changing haematological parameters in dengue viral infections, Shyek Jayad P.G. Med. Institute Lahore, Vol;24(1)Page no:3-6.

Trupti Dongre, Pragati Karmarkar. Feb 2015. Hematological Parameters and Its Utility in Dengue. A Prospective. Study. Indian J. Med. Dent. Sci., Vol;14(2) DOI: 10.9790/0853-14223134 Ver. II Page no: 31-34.

World Health Organisation. 2009. Dengue: Guidelines for Diagnosis, Treatment, Prevention and Control. New Edition, World Health Organization and TDR for research on diseases of poverty. ISBN 9789241547871 Page no: 97-106.

\section{How to cite this article:}

Krishna Murthy Sirigadha and Md. Khaleel. 2017. A Study on the Laboratory Profiles in Dengue Viral Infection. Int.J.Curr.Microbiol.App.Sci. 6(1): 387-395.

doi: http://dx.doi.org/10.20546/ijcmas.2017.601.047 